\title{
Scaling solutions of the two fluid hydrodynamic equations in a harmonically trapped gas at unitarity
}

\author{
Yan-Hua Hou ${ }^{1}$, Lev P. Pitaevskii ${ }^{1,2}$, Sandro Stringari ${ }^{1}$ \\ 1 Dipartimento di Fisica, Università di Trento and INO-CNR BEC Center, I-38123 Povo, Italy \\ 2 Kapitza Institute for Physical Problems, Russian Academy of Science, Kosygina 2, 119334 Moscow, Russia
}

(Dated: October 16, 2018)

\begin{abstract}
We prove that the two fluid Landau hydrodynamic equations, when applied to a gas interacting with infinite scattering length (unitary gas) in the presence of harmonic trapping, admit exact scaling solutions of mixed compressional and surface nature. These solutions are characterized by a linear dependence of the velocity field on the spatial coordinates and a temperature independent frequency which is calculated in terms of the parameters of the trap. Our results are derived in the regime of small amplitude oscillations and hold both below and above the superfluid phase transition. They apply to isotropic as well as to deformed configurations, thereby providing a generalization of Castin's theorem (Y. Castin, C. R. Phys. 5, 407 (2004)) holding for isotropic trapping. Our predictions agree with the experimental findings in resonantly interacting atomic Fermi gases. The breathing scaling solution, in the presence of isotropic trapping, is also used to prove the vanishing of two bulk viscosity coefficients in the superfluid phase.

PACS numbers: 67.85.Lm, 03.75.Ss, 03.75.Kk
\end{abstract}

The thermodynamic behavior of Fermi gases interacting with infinite scattering length (hereafter called unitary Fermi gases) are known to exhibit peculiar universal properties. This follows from the fact that in the unitary limit of uniform configurations the only available lengths are the interparticle distance and the thermal wavelength. Analogously the remaining energies are the thermal energy $k_{B} T$ and the Fermi energy $E_{F}=$ $\frac{\hbar^{2}}{2 m}\left(3 \pi^{2} n\right)^{2 / 3}$, where $k_{B}$ is the Boltzmann constant and $n$ is the particle density. This peculiarity makes these systems extremely interesting because their thermodynamic behavior takes a universal character, suitable to explain several physical features of quite different systems, like resonantly interacting atomic gases and neutron matter. Systematic experimental [1-4] and theoretical (see for example [5 7] and refs therein) efforts have been made to explore this universal thermodynamic behavior in the superfluid as well as in the normal phase. The concept of unitarity is not restricted to the Fermi gas and applies to Bose gases as well [8 10], although three-body recombinations cause a fast instability of these systems, making their experimental investigation problematic especially at low temperature.

The unitary Fermi gas is known to exhibit unique features also from the dynamic point of view. In particular Castin 11] has shown that exact scaling solutions are available if the system is trapped by an isotropic threedimensional harmonic potential. A remarkable example is the occurrence of an undamped radial breathing mode oscillating at the frequency $2 \omega_{h o}$, where $\omega_{h o}$ is the oscillator frequency of the harmonic potential. This result is remarkable because it concerns a strongly interacting system and its validity is not restricted to small amplitude oscillations. Furthermore it holds exactly at all temperatures irrespective of the collisional regime and the value of the mean free path. It can be regarded as the strongly interacting and quantum analog of the most famous clas- sical result derived by Boltzmann for an ideal gas trapped by an isotropic harmonic potential [12].

Similarly to the case of the Boltzmann gas, the universality of the scaling oscillation breaks down in the presence of a deformed harmonic trap. In this case no general exact result is available, unless one considers the collisional hydrodynamic regime characterized by the occurrence of fast collisions. This is the case considered in the present paper where we prove the existence of a class of scaling solutions describing small amplitude oscillations of the gas around equilibrium, characterized by a temperature independent value of the collective frequency. These solutions have been already identified in the literature at zero temperature (see [6] and references therein). Their existence is proven here at all temperatures, both below and above the critical temperature for superfluidity. For simplicity we consider an axially symmetric trapping potential

$$
V_{\text {ext }}=\frac{1}{2} m \omega_{\perp}^{2} \mathbf{r}_{\perp}^{2}+\frac{1}{2} m \omega_{z}^{2} \mathbf{z}^{2}
$$

where $\omega_{\perp}$ and $\omega_{z}$ are, respectively, the radial frequencies while $\mathbf{r}_{\perp}=x \hat{i}+y \hat{j}$, but our proof can be easily generalized to the more general case of tri-axial trapping.

We start from the two fluid hydrodynamic equations

$$
\begin{gathered}
m \partial_{t} n+\nabla \cdot \mathbf{j}=0 \\
\partial_{t} s+\nabla \cdot\left(s \mathbf{v}_{n}\right)=0 \\
m \partial_{t} \mathbf{v}_{s}=-\nabla\left(\mu+V_{e x t}\right) \\
\partial_{t} \mathbf{j}=-\nabla P-n \nabla V_{e x t},
\end{gathered}
$$

first derived by Landau [13], which describe the dynamic behavior of a gas in its superfluid phase. Equation (2) 
is the equation of continuity where $n$ is the density of the fluid and $\mathbf{j}=m\left(n_{n} \mathbf{v}_{n}+n_{s} \mathbf{v}_{s}\right)$ is the current density where we have introduced the superfluid $\left(n_{s}\right)$ and normal densities $\left(n_{n}\right)$ and the corresponding velocity fields $\mathbf{v}_{n}$ and $\mathbf{v}_{s}$. Equation (3) shows that the transport of entropy ( $s$ is the entropy density) is carried by the normal component of the fluid. Equation (4) instead fixes the equation for the superfluid velocity, governed by the chemical potential $(\mu=\mu(n, T)$ is the local value of the chemical potential, determined by the equation of state of uniform matter). Finally Eq. (5) is the Euler equation for the current fixed by the gradient of the pressure $P$ and of the external potential $V_{\text {ext }}$. Above the superfluid critical temperature the superfluid density vanishes and Eqs. (223) and (5) reduce to the usual hydrodynamic equations of a normal fluid.

In the following we prove the existence of exact solutions of the hydrodynamic equations, corresponding to the first sound like ansatz $\mathbf{v}_{n}=\mathbf{v}_{s} \equiv \mathbf{v}$ for the velocity field of the normal and superfluid components with:

$$
\mathbf{v}=\beta(t) \mathbf{r}_{\perp}+\delta(t) \mathbf{z} .
$$

Above $T_{c}$, where the superfluid density is absent, the same anstaz applies to the velocity field of the fluid. In Eq. (6) we have considered a velocity flow with symmetric behavior in the $x$ and $y$ directions, corresponding to excitations carrying angular momentum along the z-th direction $\ell_{z}=0$. The generalization of the formalism to scaling excitations carrying angular momentum $\ell_{z}= \pm 1$ and $\ell_{z}= \pm 2$ is straightforward.

The choice (6) is accompanied by the following scaling transformations for the density

$$
n(\mathbf{r}, t)=e^{2 \alpha(t)+\gamma(t)} n_{0}\left(\mathbf{r}^{\prime}\right)
$$

and for the entropy density

$$
s(\mathbf{r}, t)=e^{2 \alpha(t)+\gamma(t)} s_{0}\left(\mathbf{r}^{\prime}\right)
$$

where $\mathbf{r}^{\prime} \equiv\left(e^{\alpha(t)} x, e^{\alpha(t)} y, e^{\gamma(t)} z\right)$ is the scaled spatial variable and $n_{0}$ and $s_{0}$ are the particle density and entropy density calculated at equilibrium. The prefactor $e^{2 \alpha(t)+\gamma(t)}$ in the above equations ensures the normalization condition for the density and the conservation of total entropy. The local entropy per particle $s(\mathbf{r}) / n(\mathbf{r})$ is also conserved. At unitary the entropy density can be written in the form $s(n, T)=n f_{e}\left(T / T_{F}(n)\right)$ where $T_{F}=E_{F} / k_{B}$ is the Fermi temperature and $f_{e}$ is a universal function that can be derived by the knowledge of the equation of state (see for example [14]), but whose explicit form is irrelevant for the proof of our theorem. The ansatz (8) then requires that the ratio $T / T_{F}(n)$ should be conserved by the scaling transformation. This implies that the temperature should exhibit the position independent scaling law

$$
T(t)=e^{(2 \alpha(t)+\gamma(t)) 2 / 3} T_{0}
$$

where $T_{0}$ is the temperature of the gas at equilibrium. Finally the chemical potential, which due to dimensionality arguments can be written in the form $\mu=$
$T_{F}(n) f_{\mu}\left(T / T_{F}(n)\right)$ where $f_{\mu}$ is a dimensionless function, exhibits the following scaling behavior

$$
\mu(\mathbf{r}, t)=e^{(2 \alpha(t)+\gamma(t)) 2 / 3} \mu_{0}\left(n_{0}\left(\mathbf{r}^{\prime}\right)\right)
$$

with $\mu_{0}$ calculated at the equilibrium temperature $T_{0}$. The fact that temperature fluctuations associated with the scaling solutions are uniform in space represents a peculiar feature of these collective oscillations. It implies, in particular, that as a consequence of the thermodynamic relationship $\nabla P=s \nabla T+n \nabla \mu$ and of the ansatz (6), the equations (4) and (5) for the superfluid velocity and for the current are exactly equivalent.

We now prove that the above scaling ansatz actually corresponds to an exact solution of the hydrodynamic equations. From the equation of continuity one finds the following relationship

$$
\begin{array}{r}
{[(2 \dot{\alpha}+\dot{\gamma})+(2 \beta+\delta)] n_{0}} \\
+(\beta+\dot{\alpha}) \mathbf{r}_{\perp} \cdot \nabla_{\perp} n_{0} \\
+(\delta+\dot{\gamma}) z \nabla_{z} n_{0}=0
\end{array}
$$

which implies the identities

$$
\dot{\alpha}=-\beta, \quad \dot{\gamma}=-\delta
$$

The same conditions permit to satisfy the equation for the entropy density.

Since at equilibrium the chemical potential satisfies the condition $\nabla \mu_{0}(\mathbf{r})=-m \omega_{\perp}^{2} \mathbf{r}_{\perp}-m \omega_{z}^{2} \mathbf{z}$ (see Eq.(4)), the equation for the superfluid velocity (or, equivalently, the equation for the total current) takes the simplified form

$$
\begin{aligned}
\dot{\beta} \mathbf{r}_{\perp}+\dot{\delta} \mathbf{z}= & \left(e^{2 \alpha+(2 \alpha+\gamma) 2 / 3}-1\right) \omega_{\perp}^{2} \mathbf{r}_{\perp} \\
& +\left(e^{2 \gamma+(2 \alpha+\gamma) 2 / 3}-1\right) \omega_{z}^{2} \mathbf{z}
\end{aligned}
$$

By looking for time dependent solutions proportional to $e^{-i \omega t}$ and expanding the exponentials of Eq.(13) up to terms linear in $\alpha$ and $\gamma$, one finally derives the coupled equations

$$
\omega^{2} \alpha=\left(\frac{10}{3} \alpha+\frac{2}{3} \gamma\right) \omega_{\perp}^{2}
$$

and

$$
\omega^{2} \gamma=\left(\frac{4}{3} \alpha+\frac{8}{3} \gamma\right) \omega_{z}^{2}
$$

yielding the temperature independent result

$$
\omega^{2}=\left(\frac{5}{3}+\frac{4}{3} \lambda^{2} \pm \frac{1}{3} \sqrt{16 \lambda^{4}-32 \lambda^{2}+25}\right) \omega_{\perp}^{2}
$$

for the collective frequencies where $\lambda=\omega_{z} / \omega_{\perp}$ is the aspect ratio of the trap.

For isotropic trapping $\left(\lambda=1\right.$, i.e. $\left.\omega_{\perp}=\omega_{z} \equiv \omega_{h o}\right)$ the corresponding modes are the uncoupled monopole (breathing) mode with $\omega=2 \omega_{h o}$ and the surface quadrupole mode with $\omega=\sqrt{2} \omega_{h o}$. For highly elongated 
traps $\left(\lambda \ll 1\right.$, i.e. $\left.\omega_{z} \ll \omega_{\perp}\right)$ the two solutions are instead $\omega=\sqrt{10 / 3} \omega_{\perp}$ and $\omega=\sqrt{12 / 5} \omega_{z}$, in agreement with the results already derived in the literature at zero temperature (see for example [15, 16]).

The above results provide a generalization of the theorem of [11], where it was shown that for isotropic trapping the monopole breathing mode of the unitary Fermi gas oscillates with frequency $\omega=2 \omega_{h o}$, independent of temperature. Differently from the case of [11] our results hold, however, only in the dissipationless hydrodynamic regime and in the limit of small amplitude oscillations. The temperature independence of the frequency of the scaling solutions of the unitary Fermi gas has been already confirmed in experiments [17, 18].

The scaling solutions discussed above are characterized by temperature variations and by an axial velocity field $v_{z}$ independent of the radial coordinates $x$ and $y$. These are the conditions required, in general, to apply the 1D hydrodynamic equations in highly elongated configurations (see [14, 19]) which have been recently successfully applied to describe the experimental results of [18]. In general these $1 \mathrm{D}$ like conditions are ensured by the effective role of the thermal conductivity and of the viscosity which cause the absence of gradients in the radial direction and are favored by the presence of a tight radial confinement. In the case of the low frequency oscillations considered in the present work the absence of radial gradients is automatically ensured by the form of the scaling transformation. This explains, in particular, why the frequency $\omega=\sqrt{12 / 5} \omega_{z}$ of the axial breathing mode, here derived in a 3D framework in the highly elongated limit $\omega_{z} \ll \omega_{\perp}$, coincides with the predictions of the 1D hydrodynamic equations [14].

Let us also mention that the proof of the temperature independence of the frequency of the scaling solutions can be derived also for the $\ell_{z}= \pm 1$ and $\ell_{z}= \pm 2$ excitations characterized by velocity fields proportional to $\nabla[z(x \pm i y)]$ and $\nabla(x \pm i y)^{2}$, respectively and yielding the values $\sqrt{\omega_{\perp}^{2}+\omega_{z}^{2}}$ and $\sqrt{2} \omega_{\perp}$ for the corresponding frequencies. Differently from the $\ell_{z}=0$ solutions discussed above the results for the $\ell_{z}= \pm 1$ and $\ell_{z}= \pm 2$ are not restricted to the unitary Fermi gas, but simply require the applicability of the hydrodynamic equations, being solutions characterized by divergency free velocity fields (surface excitations). They hold in particular for both Fermi and Bose gases in the presence of harmonic trapping.

Let us finally discuss a non trivial implication of the scaling solutions concerning the behavior of the bulk viscosity coefficients. According to Khalatnikov [20] the entropy production per unit volume associated with a hydrodynamic flow is $R / T$, where $R$ is the so called dissipative function in the superfluid phase, defined by

$$
\begin{aligned}
R= & \frac{1}{2} \eta\left(\partial_{k} v_{n i}+\partial_{i} v_{n k}-\frac{2}{3} \delta_{i k} \nabla \cdot \mathbf{v}_{n}\right)^{2} \\
& +2 \zeta_{1} \nabla \cdot \mathbf{v}_{n} \nabla \cdot m n_{s}\left(\mathbf{v}_{s}-\mathbf{v}_{n}\right)+ \\
& +\zeta_{2}\left(\nabla \cdot \mathbf{v}_{n}\right)^{2}+\zeta_{3}\left[\nabla \cdot m n_{s}\left(\mathbf{v}_{s}-\mathbf{v}_{n}\right)\right]^{2} \\
& +(\kappa / T)(\nabla T)^{2} .
\end{aligned}
$$

In the above equation $\eta$ is the shear viscosity, $\zeta_{1}, \zeta_{2}$ and $\zeta_{3}$ are the three bulk viscosity coefficients appearing in the superfluid phase, while $\kappa$ is the thermal conductivity. For the scaling modes discussed in the present paper $\nabla T=0$ and the velocity fields for the normal and superfluid components coincide. Furthermore for the monopole breathing mode in an isotropic trap one has $\mathbf{v}_{n}=\mathbf{v}_{s}=\beta \mathbf{r}$ and the first term, proportional to the shear viscosity, identically vanishes. It follows that in this case only the term with $\zeta_{2}$ survives. However, according to Castin theorem [1] the dissipation associated with the breathing oscillation must be zero and we then conclude that $\zeta_{2}=0$. On the other hand the positiveness of $R$ implies that $\zeta_{1}^{2} \leq \zeta_{2} \zeta_{3}$. Thus also $\zeta_{1}$ must vanish, the only surviving bulk viscosity coefficient being $\zeta_{3}$. Above $T_{c}$, where one can introduce only one bulk viscosity term, the viscosity coefficient $\zeta$ should be also zero in order to ensure the absence of dissipation. The same results, concerning the value of the bulk viscosity coefficients, were previously derived by Son [21] using different considerations. Actually, our derivation provides a simple foundation to Son's heuristic argumentation.

\section{Acknowledgments}

This work has been supported by ERC through the QGBE grant.
[1] L. Luo and J. E. Thomas, J. Low Temp Phys, 154, 1 (2009).

[2] S. Nascimbène, N. Navon, K. J. Jiang, F. Chevy and C. Salomon, Nature 463, 1057 (2010).

[3] M. Horikoshi, S. Nakajima, M. Ueda and T. Mukaiyama, Science 327, 442 (2010).

[4] Mark J. H. Ku, Ariel T. Sommer, Lawrence W. Cheuk and Martin W. Zwierlein, Science 335, 563 (2012).

[5] Ho, T.-L., Phys. Rev. Lett. 92, 090402 (2004).

[6] S. Giorgini and L. P. Pitaevskii and S. Stringari, Rev.
Mod. Phys. 80, 1215 (2008).

[7] Immanuel Bloch, Jean Dalibard, and Wilhelm Zwerger, Rev. Mod. Phys. 80, 885 (2008).

[8] W. Li and T.-L. Ho, Phys. Rev. Lett. 108, 195301 (2012).

[9] B. S. Rem, A. T. Grier, I. Ferrier-Barbut, U. Eismann, T. Langen, N. Navon, L. Khaykovich, F. Werner, D. S. Petrov, F. Chevy, and C. Salomon, arXiv:1212.5274 (2012).

[10] J. J. R. M. van Heugten and H. T. C. Stoof, arXiv:1302.1792 (2013). 
[11] Castin Y, C. R. Physique 5407 (2004).

[12] C. Cercignani, The Boltzmann equation and its applications. Springer Verlag, New York, 1988.

[13] L. D. Landau, J. Phys. USSR 5, 71 (1941).

[14] Y.-H. Hou, L. Pitaevskii, S. Stringari, arXiv:1301.4419 (2013).

[15] M. Amoruso, I. Meccolia, A. Minguzzi, and M. P. Tosi, Eur. Phys. J. D 7, 441 (1999).

[16] S. Stringari, Europhys. Lett. 65, 749 (2004).

[17] A. Altmeyer, S. Riedl, C. Kohstall, M. J. Wright, R. Geursen, M. Bartenstein, C. Chin, J. Hecker Denschlag, and R. Grimm, Phys. Rev. Lett. 98, 040401 (2007).

[18] M. K. Tey, L. A. Sidorenkov, E. R. S. Guajardo, R. Grimm, M. J. H. Ku, M. W. Zwierlein, Y.-H. Hou, L. Pitaevskii, and S. Stringari Phys. Rev. Lett. 110, 055303 (2013).

[19] G. Bertaina, L. Pitaevskii and S. Stringari, Phys. Rev. Lett. 105, 150402 (2010)

[20] I. M. Khalatnikov, An Introduction to the Theory of Superfluidity (Benjamin, New York, 1965).

[21] D. T. Son, Phys. Rev. Lett. 98, 020604 (2007). 